\title{
Wide-field digital imaging based telemedicine for screening for acute retinopathy of prematurity (ROP). Six-year results of a multicentre field study
}

\author{
Birgit Lorenz • Katerina Spasovska • Heike Elflein • \\ Nico Schneider
}

Received: 3 August 2008 /Revised: 17 March 2009 / Accepted: 24 March 2009 / Published online: 22 May 2009

(C) The Author(s) 2009. This article is published with open access at Springerlink.com

\begin{abstract}
Objective To report on a 6-year experience with wide-field digital imaging based telemedicine (WFDI telemedicine) to reduce the risk for blindness from retinopathy of prematurity (ROP).

Methods Wide-angle digital fundus cameras (RetCam 120, Massie Lab, Pleasanton, CA, USA) were installed in five neonatal intensive care units (NICUs) in Germany. All prematures at risk were screened with WFDI, and the local ophthalmologists were asked to continue binocular indirect ophthalmoscopy (BIO) according to the German guidelines.
\end{abstract}

Support: Bayerische Sparkassenstiftung, München, Germany; Bayerische Landesstiftung, München, Germany; Deutsche Forschungsgemeinschaft (DFG Lo 457/4-1,2), and Iris Medical, Mountain View, CA, USA

The authors do not have any financial interest in the RetCam120 or Massie Laboratories, Pleasanton, CA 94588, USA or Clarity Medical Systems Inc, Pleasanton, CA 94588, USA

C: (925)-519-6655, www.claritymsi.com

Parts of the contents were presented at the Annual Meetings 2003 and 2007 of the Association for Research in Vision and Ophthalmology ARVO, at the Annual Meeting of the European Paediatric Ophthalmological Society EPOS 2003, at the Annual Meeting 2004 of the German Ophthalmological Society DOG, and at the World Ophthalmology Congresses WOC 2006 and 2008

B. Lorenz $(\bowtie) \cdot$ N. Schneider

Department of Ophthalmology,

Justus-Liebig-University Giessen, Universitaetsklinikum Giessen and Marburg GmbH Giessen Campus,

Friedrichstrasse 18,

35385 Giessen, Germany

e-mail: birgit.lorenz@uniklinikum-giessen.de

B. Lorenz $\cdot$ K. Spasovska $\cdot$ H. Elflein $\cdot$ N. Schneider

Department of Paediatric Ophthalmology, Strabismology and

Ophthalmogenetics, Regensburg University Medical Centre,

University of Regensburg,

Regensburg, Germany
Image data were coded and transferred to the Reading Centre in Regensburg. Image evaluation and additional BIO of infants with suspected treatment-requiring ROP (STRROP i.e. threshold ROP zone II, prethreshold ROP zone I (type-1 ROP according to ETROP), and ROP possibly requiring treatment but not reliably classifiable from the images) were performed by paediatric ophthalmologists at the Reading Centre. ROP was classified following ICROP, ETROP, and revised ICROP criteria. Outcome measures were incidence of clinically relevant ROP (CR-ROP, i.e. any ROP up to mid-peripheral zone III, $\leq$ stage $3+$ ),

K. Spasovska

Department of Ophthalmology,

Regensburg University Medical Centre,

University of Regensburg,

Regensburg, Germany

H. Elflein

Department of Ophthalmology,

Johannes Gutenberg-Universitaet,

Mainz, Germany 
sensitivity to detect STR-ROP, and positive predictive value to detect treatment-requiring ROP (TR-ROP).

Results In total, 1,222 prematures at risk were screened (mean BW $1395 \mathrm{~g}, \mathrm{SD} \pm 507 \mathrm{~g}$; mean GA $30 \mathrm{wks}, \mathrm{SD} \pm 3 \mathrm{wks})$. The overall incidence of CR-ROP was $27.6 \%(71.8 \%$ mild $=$ stage 1 to 3 without plus disease, $15.7 \%$ prethreshold=type- 1 ROP according to ETROP, $12.5 \%$ threshold according to ICROP). Zone I disease was present in 3.3\%, zone II disease in $76.5 \%$, and zone III disease in 20.2\%. According to ETROP, 95 infants were type- 1 or type- 2 ROP; $67.4 \%$ type- 1 ROP, and $32.6 \%$ type-2 ROP. Of all 1,222 infants, 3.5\% received treatment. Following ETROP (not applied in the study), 5.3\% would have been treated. The sensitivity for detecting STR-ROP was $100 \%$, and the positive predictive value for TR-ROP $82.4 \%$ $(28 / 34)$ at the time of the first referral ( 28 infants, $\leq$ stage $3+$ in zone I or II).

Conclusion All TR-ROP was detected in time, showing the potential of our telemedical screening program. The overall incidence of CR-ROP was comparable to ROP incidences reported in other West European countries.

Keywords Retinopathy of prematurity (ROP) .

Wide-field digital imaging (WFDI) · RetCam 120 .

Telemedicine $\cdot$ Sensitivity $\cdot$ Positive predictive value

\section{Introduction}

Retinopathy of prematurity (ROP) is a potentially blinding disease that occurs in premature infants, and affects the postnatal maturation of the retinal blood vessels. Many significant risk factors have been identified, including various growth factors [1-13]. The proportion of childhood blindness caused by ROP now varies from $8 \%$ in highincome countries to $40 \%$ in middle-income countries, and up to $60 \%$ in some regions $[14,15]$.

In 1988, the "Cryotherapy for ROP" study showed that cryotherapy at threshold disease (stage $3+$ in zone I or II, extraretinal proliferations in 5 contiguous or 8 clock hours in total according to ICROP [16]) halved the incidence of retinal detachment and, hence, adverse outcome. If left untreated, as many as $50 \%$ of the children had an unfavourable outcome [17]. Presently, the preferred treatment modality of threshold ROP is laser photocoagulation, which decreases the risk of retinal detachment to only a few percent $[18,19]$. In high-income countries, today about $5 \%$ of infants with a birth weight below $1250 \mathrm{~g}$ develop threshold disease [20]. Timing appears crucial in terms of treatment success. Once threshold disease is diagnosed, delay in treatment has the risk of leading to more severe ROP, i.e. "suprathreshold disease", and therefore worsens the prognosis [21]. Following the more recent Early Treatment of ROP (ETROP) study [22], the criteria and indications for treatment of ROP, particularly the optimum time for treatment, are under discussion in many countries. According to ETROP, i.e. earlier treatment of type-1 ROP, a better overall ROP outcome is advocated [23].

Expert screening by paediatric ophthalmologists or retina specialists for repeated exams to detect treatment-requiring disease according to the evolving definitions is crucial. Such expert screening is not always available, although detailed screening guidelines have been provided in various countries and updated recently [24-30]. In Germany, ROP screening in the NICUs is frequently provided by general ophthalmologists with different degrees of experience with the disease. This is due to the fact that there are at least four times more NICUs than university sites where treatment is usually performed. A typical German NICU has 30-60 preterm babies at risk for ROP per year. With the incidence of threshold disease of about $5 \%$ in babies with a birth weight below $1250 \mathrm{~g}$ [31], each NICU will have, on average, two to three preterm babies at threshold per year (with spontaneous regression in 50\%). Hence, the consulting ophthalmologist-sometimes there are several per NICU - may not gain sufficient experience in evaluating the disease. In Germany, direct pension costs for a blind person (independent of their income) are about 300,000€ based on the current rates and calculated for a period of 50 years. They do not include specific costs for education of the blind and specialised equipment for work. Consequently, even a few failed screenings or treatments present a compelling economic reason to support screening and treatment programs when multiplied by the high cost of failure.

Telemedicine combined with WFDI offers the opportunity for improved ROP screening by expert evaluation of the images independent of the screening site. The RetCam120, a retinal wide-field digital imaging system, allows documentation of large parts of the retina of the alert infant within minutes. Several studies [32-45], have now evaluated the value of WFDI in screening for ROP. In our prospective field study we have used, since 2001, the RetCam120 in five Bavarian NICUs for ROP screening of infants at risk scheduled according to the German national guidelines. Four of these NICUs were peripheral sites, with ROP screening performed by general ophthalmologists. All images were transferred to the Reading Centre at the University of Regensburg for evaluation. In a subset of infants, both WFDI and binocular indirect ophthalmoscopy BIO were performed by masked examiners. For all other infants, the local ophthalmologists were asked to continue $\mathrm{BIO}$ as requested by the German guidelines, but in fact did use BIO at their discretion. Our program was targeted at detection of clinically relevant ROP (CR-ROP, i.e. any ROP up to mid-peripheral zone III) with WFDI and 
telemedicine, to schedule adequate follow-up examinations, and to identify suspected treatment-requiring ROP (STRROP, definition see below) and thereby ideally eliminate late referrals. Our program was not targeted at reduction of ophthalmic examinations [46].

\section{Material and methods}

\section{Patients}

The study followed the Tenets of the Declaration of Helsinki (revised version, 1989) and was approved by the Ethics Committee of the University of Regensburg (00/ 190). Informed consent was obtained from all parents participating in the study.

In February 2001, RetCam120s were installed in five NICUs in East Bavaria covering a distance of about $200 \mathrm{~km}$, and every RetCam120 user at that time received instruction in the use of the instrument from an ophthalmic photographer experienced in the use of the RetCam (Leslie MacKeen, Hospital for Sick Children, Toronto, Canada). Starting from that date, all prematures at risk were examined with the RetCam 120 following the German screening guidelines as to timing of examinations, gestational age (GA), and birth weight (BW) (Table 1) [29]. At the same time, all participants were asked to continue conventional screening with BIO.

\section{Examination protocol}

Imaging was performed by general ophthalmologists in the four peripheral NICUs, and by paediatric ophthalmologists of the Reading Centre in the NICU in Regensburg. The ophthalmologists were directed to begin their examinations at a chronological age (CA) of 3642 days, but not before a postmenstrual age (PMA) of 31 weeks (Table 1).

In the peripheral NICUs, only one examiner at the time did the examinations. Therefore, an objective comparison of BIO and WFDI did not appear feasible there. However, all examiners were instructed to inform the Reading Centre in

Table 1 German guidelines for screening for acute ROP [29]*

All babies with a $\mathrm{GA}<32$ wks and/or a $\mathrm{BW}<1,501 \mathrm{~g}$

Babies with a $\mathrm{GA}<36$ wks and artificial oxygen

ventilation for $>3$ days.

First examination 36 to 42 days PNA, not before 31 wks PMA

$G A$ gestational age, $B W$ birth weight, $P N A$ postnatal age, $P M A$ postmenstrual age

*The German Guidelines have been revised recently [30]. They have remained unchanged for the parameters given in the table case of discrepancies of their estimation of ROP with BIO and the results of image evaluation at the Reading Centre.

In 2002 and 2003, in a subset of 63 consecutive examinations (41 infants) WFDI was performed in the NICU of the Department of Paediatrics in Regensburg, by a senior resident, and in parallel conventional BIO by a paediatric ophthalmologist highly experienced with all stages of acute ROP (H.E.). Both examiners classified their findings from either WFDI or BIO, without knowing the result obtained with the alternative method. The images were read again in 2008 by the senior investigator (B.L.) who was masked as to the $\mathrm{BIO}$ results.

Examinations were performed after timely administration of atropine $0.1 \%$ (custom-made) and phenylephrine $2 \%$ (Neosynephrin-POS ${ }^{\circledR} 2 \%$ ) eye drops for complete mydriasis, and oxybuprocain (Benoxinat ${ }^{\circledR}$ ) eye drops for local anaesthesia of the cornea and conjunctiva (in the authors'experience, atropine $0.1 \%$ is particularly useful for obtaining good mydriasis over a period of several hours, which eliminates the problem of small pupils if the examination was not done at the scheduled hour). A sterile wire lid speculum (Barraquer for premature infants, Geuder, Heidelberg, Germany) was used to keep the eyelids open, as this speculum is the least spaceconsuming, and therefore allows good imaging even in the smallest infants.

The ophthalmologists were instructed to take six images per eye with the ROP lens that visualizes $130^{\circ}$ : five images of the retina of each eye in a contact procedure (one posterior pole image and four more peripheral images to cover most of the retina), and one non-contact image of the anterior part of the eye for evaluating an eventual hyperaemia of the iris and persistent tunica vasculosa lentis as a sign of acute ROP (from January 2002). During the course of the study, it became clear that eight images from the peripheral retina were better suited to imaging the whole circumference. This is now the recommended standard set (Fig. 1). Follow-up examinations were scheduled by the Reading Centre according to the pathology seen and following the German guidelines [29], i.e. weekly in the case of acute ROP in zone I and II if not yet considered STR-ROP, and every 2 weeks in the absence of ROP but still incomplete vascularisation, in case of mild zone III disease, or in the case of beginning regression of any ROP. Therapy, if required, was performed on site or at the Reading Centre, according to availability of prolonged anaesthesia in the peripheral NICUs. The treatment decision was always based on an additional BIO exam by a specialist at the Reading Centre (senior investigator B.L., or an experienced fellow) once STR-ROP had been diagnosed. STR-ROP was defined as threshold ROP in zone II, or prethreshold in zone $\mathrm{I}=$ type-1 ROP, or 


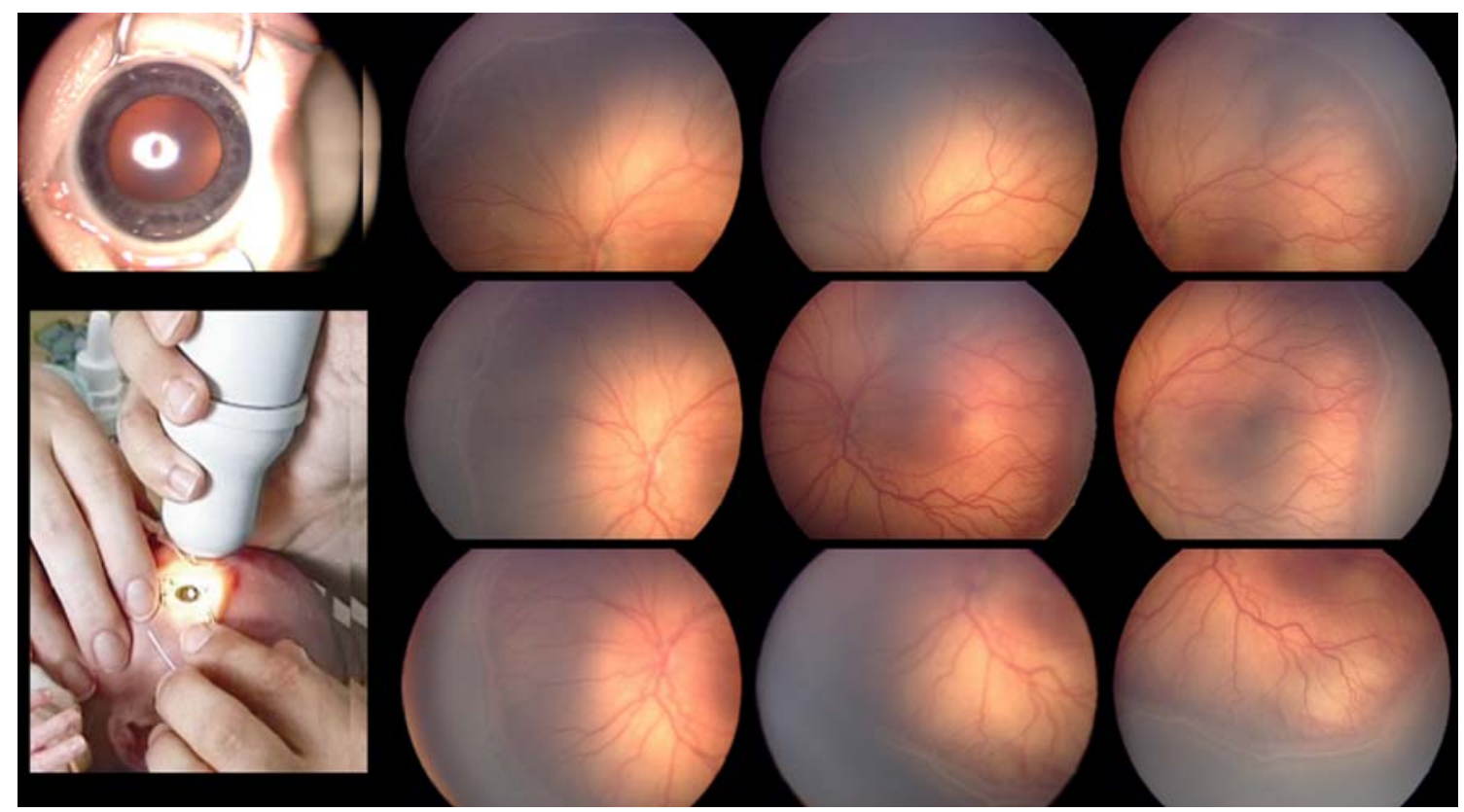

Fig. 1 Standard image set and view of camera (GA 26weeks, BW $710 \mathrm{~g})$ ROP $3+$, central zone 2. Iris hyperemia and some dilated remnants of the tunica vasculosa lentis (top left). Shown are

possibly treatment-requiring ROP that could not be reliably classified from the images. The main reasons for classification issues were incomplete imaging of the whole circumference and/or doubts as to the degree of extraretinal neovascularisation. WFDI was continued until vascularisation was estimated to be complete i.e. when retinal vessels were seen in mid-peripheral zone III, or any ROP had regressed spontaneously or STR-ROP had developed. The extreme periphery, i.e. the ora serrata, was not imaged with WFDI.

\section{Reading of digitized images}

Images from the NICU in Regensburg were evaluated by paediatric ophthalmologists experienced in reading the images, and in the case of any pathology also by the senior investigator (B.L.).

Image data received from the RetCam120 examinations outside Regensburg were sent via ISDN (phone line) to the Reading Centre without any personal data to assure data safety. The reader was also blinded as to the result of the exam in the peripheral NICU. To assign the images to the respective infants, a coding system was used consisting of 8 digits (Fig. 2). Personal and paediatric data of the infants were sent anonymously via email or fax to the Reading Centre using the same code. The images were evaluated by experienced paediatric ophthalmologists the same day or the day after, and in the case of any pathology also by the senior investigator (B.L.). These evaluations and also recommendations as to control examinations onsite or at
RetCam120 images of the left eye. Bottom left panel shows the camera in non-contact mode to visualize the anterior segment (from [54])

the Reading Centre were sent back via email to the referring NICU.

Complete imaging with the technology available today is not feasible in all instances. This does not represent a real drawback, as the important issue in the context of screening is to determine whether there is indication of either immature retina or any pathology that may progress to treatment-requiring ROP, both warranting follow-up examinations. To overcome the present limitations and yet get the necessary information, assessment of the images included evaluation of: (1) presence of dilated vessels of a persistent tunica vasculosa lentis and/or iris hyperaemia, (2) extent of visible retinal vascularisation, (3) shape and diameter of retinal vessels at the posterior pole, i.e. vasoconstriction or plus disease, (4) shape, degree of arborisation and diameter of retinal vessels in the peripheral images or at the border of clear visibility, and (5) completeness of imaging as to zone and clock hours. These additional factors were used for definitive classification and to schedule follow-up examinations or eventually refer infants for BIO because of STR-ROP.

\section{$01 \mathrm{D} 00301$}

Fig. 2 Example of coding system to ensure data security in the Telemedicine Project. This child has been first examined in 2001 (01) in the NICU in Deggendorf (d) as the 3rd child examined that year at the NICU in Deggendorf (003). The last two digits 01 indicate that this was the first examination of this child 
Table 2 Baseline characteristics of all patients screened and of patients with ROP

\begin{tabular}{lll}
\hline & Screened $(n=1,222)$ & ROP $(n=337)$ \\
\hline Gender & & \\
Male [\%] & $662[54.2]$ & $172[51.1]$ \\
Female [\%] & $560[45.8]$ & $165[48.9]$ \\
Birth weight (BW) & $n=1,215^{*}$ & $n=335^{*}$ \\
Mean [g] & 1,395 & 1,047 \\
Range [g] & $410-4,780$ & $410-2,135$ \\
SD [g] & 507 & 313 \\
$<750$ g [g] & $77[6.3]$ & $61[18.2]$ \\
$750-999$ g [\%] & $198[16.3]$ & $110[32.8]$ \\
$1,000-1,249$ g [\%] & $215[17.7]$ & $82[24.5]$ \\
$1,250-1,500$ g [\%] & $343[28.2]$ & $58[17.3]$ \\
$>1,500$ g [\%]\# & $382[31.5]$ & $24[7.2]$ \\
Gestational age (GA) & $n=1,221 *$ & $n=336 *$ \\
Mean [wks] & 30 & 28 \\
Range [wks] & $22-40$ & $22-36$ \\
SD (wks) & 3 & 2 \\
$<27$ wks [\%] & $140[11.5]$ & $106[31.6]$ \\
$27-31$ wks [\%] & $778[63.7]$ & $207[61.6]$ \\
$>31$ wks [\%] & $303[24.8]$ & $23[6.8]$ \\
BW and GA & $n=1,215$ & $n=335$ \\
$<32$ wks, <1,501 g & $722[59.4 \%]$ & $297[88.7 \%]$ \\
$<32$ wks, >1,500 g & $191[15.7 \%]$ & $15.5 \%]$ \\
$>31$ wks, <1,501 g & $111[9.1 \%]$ & {$[2.7 \%]$} \\
$>31$ wks, >1,500 g & $191[15.7 \%]$ & \\
\hline
\end{tabular}

*Numbers do not correspond to the overall numbers in all instants, as in some infants, data on BW or GA is missing (in 1 infant, both values were lost).

\# The remaining $31 \%$ had one or more other risk factors for developing ROP

\section{Results}

Demographic data

From $2 / 2001$ to $12 / 2006,2,444$ eyes of 1,222 preterm babies were examined. Of these, 913 infants were examined in the four NICUs outside Regensburg, and 309 were patients of the NICU in Regensburg. Babies with a gestational age (GA) $>32$ completed gestational weeks had a birth weight $(\mathrm{BW}) \leq 1500 \mathrm{~g}$; babies with a $\mathrm{BW}>1500 \mathrm{~g}$ did not have 32 completed gestational weeks. In addition, there were a few preterm babies $(\mathrm{GA}<37$ completed gestational weeks), who had received oxygen for more than 3 days and were therefore screened for ROP. Detailed data are given in Table 2.

Image analysis

The number of RetCam120 images taken per session and transmitted to the Reading Centre varied widely from one to 60 per eye (mean ten), despite the instruction given to all examiners to take nine images per eye. One image was the exception; it was always from the posterior pole and usually at the first exam in very tiny infants. Image quality was highly variable between infants with different degrees of maturity and compliance, between the various NICUs, and also between different examiners. In total, 3,230 image sets (6,460 eyes) were taken, of which $97.6 \%(3,151 / 3,230)$ were of high enough quality to be evaluated as to (1) degree of retinal maturity, (2) retina at risk for progression to STRROP within 1 or 2 weeks, or (3) STR-ROP within days. Only $2.4 \%(79 / 3,230)$ of image sets could not be classified according to these criteria; the majority of them had been taken in the peripheral NICUs. Repeat examinations of

Table 3 Prevalence of ROP in dependence on GA in 335 infants

\begin{tabular}{|c|c|c|c|c|c|c|c|c|}
\hline GA & $<36$ wks (all infants screened*) & $<26$ wks & 26wks & $27 \mathrm{wks}$ & $28 w k s$ & $29 \mathrm{wks}$ & 30wks & $31 \mathrm{wks}$ \\
\hline Any stage of ROP & $\begin{array}{l}335 / 1,191 \\
(28.1 \%)\end{array}$ & $\begin{array}{l}58 / 71 \\
(81.7 \%)\end{array}$ & $\begin{array}{l}48 / 69 \\
(69.6 \%)\end{array}$ & $\begin{array}{l}56 / 95 \\
(58.9 \%)\end{array}$ & $\begin{array}{l}54 / 107 \\
(50.5 \%)\end{array}$ & $\begin{array}{l}47 / 136 \\
(34.6 \%)\end{array}$ & $\begin{array}{l}33 / 197 \\
(16.8 \%)\end{array}$ & $\begin{array}{l}17 / 243 \\
(7.0 \%)\end{array}$ \\
\hline STR-ROP & $\begin{array}{l}46 / 1,191 \\
(3.9 \%)\end{array}$ & $\begin{array}{l}20 / 71 \\
(28.2 \%)\end{array}$ & $\begin{array}{l}14 / 69 \\
(20.3 \%)\end{array}$ & $\begin{array}{l}4 / 95 \\
(4.2 \%)\end{array}$ & $\begin{array}{l}4 / 107 \\
(3.7 \%)\end{array}$ & $\begin{array}{l}3 / 136 \\
(2.2 \%)\end{array}$ & $\begin{array}{l}0 / 197 \\
(0.0 \%)\end{array}$ & $\begin{array}{l}1 / 243 \\
(0.4 \%)\end{array}$ \\
\hline TR-ROP & $\begin{array}{l}42 / 1,191 \\
(3.5 \%)\end{array}$ & $\begin{array}{l}20 / 71 \\
(28.2 \%)\end{array}$ & $\begin{array}{l}10 / 69 \\
(14.5 \%)\end{array}$ & $\begin{array}{l}4 / 95 \\
(4.2 \%)\end{array}$ & $\begin{array}{l}4 / 107 \\
(3.7 \%)\end{array}$ & $\begin{array}{l}3 / 136 \\
(2.2 \%)\end{array}$ & $\begin{array}{l}0 / 197 \\
(0.0 \%)\end{array}$ & $\begin{array}{l}1 / 243 \\
(0.4 \%)\end{array}$ \\
\hline Zone I & $\begin{array}{l}11 / 42 \\
(26.2 \%)\end{array}$ & $\begin{array}{l}8 / 20 \\
(40.0 \%)\end{array}$ & $\begin{array}{l}3 / 10 \\
(30.0 \%)\end{array}$ & $\begin{array}{l}0 / 4 \\
(0.0 \%)\end{array}$ & $\begin{array}{l}0 / 4 \\
(0.0 \%)\end{array}$ & $\begin{array}{l}0 / 3 \\
(0.0 \%)\end{array}$ & $\begin{array}{l}0 / 0 \\
(0.0 \%)\end{array}$ & $\begin{array}{l}0 / 1 \\
(0 \%)\end{array}$ \\
\hline Zone II & $\begin{array}{l}31 / 42 \\
(73.8 \%)\end{array}$ & $\begin{array}{l}12 / 20 \\
(60.0 \%)\end{array}$ & $\begin{array}{l}7 / 10 \\
(70.0 \%)\end{array}$ & $\begin{array}{l}4 / 4 \\
(100 \%)\end{array}$ & $\begin{array}{l}4 / 4 \\
(100 \%)\end{array}$ & $\begin{array}{l}3 / 3 \\
(100 \%)\end{array}$ & $\begin{array}{l}0 / 0 \\
(0.0 \%)\end{array}$ & $\begin{array}{l}1 / 1 \\
(100 \%)\end{array}$ \\
\hline
\end{tabular}

GA gestational age; STR-ROP suspected treatment-requiring ROP; TR-ROP treatment-requiring ROP cases are indicated in bold letters

The column $<36$ wks gives the total numbers of all infants screened and with documented GA. *As for some babies the gestational age was not documented, the total number of babies examined differs in Table 2. 
Table 4 Prevalence of ROP in dependence on BW in 335 infants

\begin{tabular}{llllll}
\hline BW & $<4,780 \mathrm{~g}$ (all infants screened*) & $<750 \mathrm{~g}$ & $750 \mathrm{~g}$ to $<1,000 \mathrm{~g}$ & $1,000 \mathrm{~g}$ to $<1,250 \mathrm{~g}$ & $1,250 \mathrm{~g}$ to $1,500 \mathrm{~g}$ \\
\hline Any stage of ROP & $335 / 1,215$ & $61 / 77$ & $110 / 198$ & $82 / 215$ & $58 / 343$ \\
& $(27.6 \%)$ & $(79.2 \%)$ & $(55.6 \%)$ & $(38.1 \%)$ & $(16.9 \%)$ \\
STR - ROP & $46 / 1,215$ & $21 / 77$ & $21 / 198$ & $2 / 215$ & $(0.6 \%)$ \\
& $(3.8 \%)$ & $(27.3 \%)$ & $(10.6 \%)$ & $(0.9 \%)$ & $2 / 343$ \\
TR - ROP & $42 / 1,215$ & $20 / 77$ & $18 / 198$ & $2 / 215$ & $(0.6 \%)$ \\
& $(3.5 \%)$ & $(26.0 \%)$ & $(9.1 \%)$ & $(0.9 \%)$ & $0 / 2$ \\
Zone I & $11 / 42$ & $7 / 20$ & $4 / 18$ & $0 / 2$ & $(0 \%)$ \\
Zone II & $(26.2 \%)$ & $(35.0 \%)$ & $(22.2 \%)$ & $(0 \%)$ & $2 / 2$ \\
& $31 / 42$ & $13 / 20$ & $14 / 18$ & $(100 \%)$ & $(100 \%)$ \\
\hline
\end{tabular}

$B W$ birth weight; STR-ROP suspected treatment requiring ROP; TR-ROP treatment-requiring ROP cases are indicated in bold letters. The column $<4780 \mathrm{~g}$ gives the total numbers of all infants screened and with documented birth weight. *As for some babies BW was not documented, the total numbers of babies examined differs in Table 2

those 79 sets one week later allowed classification, and did not show any STR-ROP. The number of examinations per infant ranged from one to 27 (including follow-up examinations after treatment), and was 2.6 on average. The number of examinations for the 46 infants with STRROP ranged from four to 27 , and was 4.6 on average.

ROP stages detected with the RetCam 120

Detailed ROP findings are listed in Tables 3 and 4. In total, $27.6 \%$ of the 1222 premature babies (337 infants) were diagnosed with CR-ROP. Of those, 71.8\% (242/337) were mild, i.e. ROP stage 1 to 3 without plus up to mid-peripheral zone III, 15.7\% (53/337) prethreshold type-1 ROP in zone I (according to ETROP), and $12.5 \%$ (42/337) threshold in zone II (according to Cryo-ROP). Zone I disease was present in $3.3 \%(11 / 337)$, zone II disease in $76.5 \%$ (258/337), and zone III disease in 20.2\% (68/337). Forty-six babies developed a more severe ROP stage i.e. STR-ROP, making a final treatment decision by a specialist $\mathrm{BIO}$ examination necessary: 34 were from peripheral NICUs, and 12 from the local NICU (Table 5). All STR-ROP stages were detected with the RetCam 120 , i.e. the sensitivity was $100 \%$, as none of the local ophthalmologists had reported a higher stage (it is however unknown whether the local ophthalmologists had detected all pathology diagnosed with WFDI at the Reading Centre). The paediatric data of the babies with STR-ROP are listed in Table 5. Following the ETROP classification done retrospectively for all children screened since 2001, 95 were type-1 or type-2 ROP: $67.4 \%$ (64/95) type-1, and 32.6\% (31/95) type-2 ROP. All 12 infants from the local NICU and 30/34 infants from the peripheral NICUs were treated.

To evaluate the positive predictive value as to treatment-requiring ROP (TR-ROP), only the 34 infants with STR-ROP from the peripheral NICUs were considered. In those 34 infants, specialist BIO exams were performed to ultimately determine the need for treatment. Twenty-eight infants were treated at the first referral, two infants after further follow-up examinations, and in four
Table 5 Paediatric data of infants with suspected treatmentrequiring ROP making a final treatment decision by BIO necessary (only infants screened in the 4 peripheral NICUs)

$B I O$ binocular indirect ophtalmoscopy; STR-ROP suspected treatment-requiring ROP; $T R$ $R O P$ treatment-requiring $\mathrm{ROP}$

\begin{tabular}{llll}
\hline & STR-ROP $(n=34)$ & TR-ROP $(n=30)$ & Spontaneous regression $(n=4)$ \\
\hline Male [\%] & $19[55.9]$ & $15[50.0]$ & $4[100.0]$ \\
Female [\%] & $15[44.1]$ & $15[50.0]$ & 0.0 \\
Birth weight & & & \\
Mean [g] & 805 & 799 & 775 \\
Range [g] & $440-1,495$ & $440-1,495$ & $670-850$ \\
SD [g] & 203 & 229 & 82 \\
Gestational age & & & 26 \\
Mean (wks) & 26 & 26 & 26 \\
Range [wks] & $22-31$ & $22-29$ & 0 \\
SD (wks) & 2 & 2 & \\
\hline
\end{tabular}



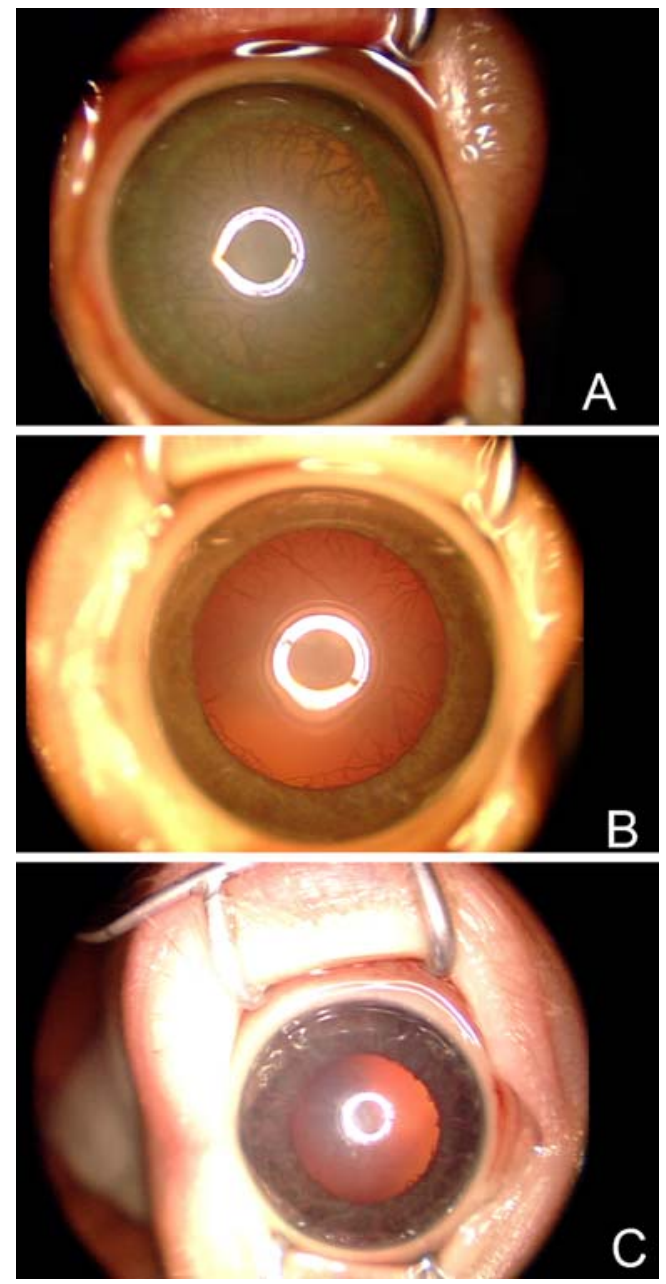

Fig. 3 Examples of iris at the time of treatment-requiring ROP. A Almost complete tunica vasculosa lentis with massive vessel dilatation. B Partial tunica vasculosa lentis, clearly hyperaemic. C Only minimal tunica vasculosa lentis. Severe engorgement of the iris vessels

infants the disease regressed spontaneously. The overall positive predictive value for TR-ROP was $88.2 \%$ : $82.4 \%$ at the first referral, and $88.2 \%$ at further follow-up.

\section{Significance of evaluating the anterior segment}

Starting from January 2002, imaging of the anterior segment was added to the protocol. In all eyes with significant ROP for which we had readable images, a dilated persistent tunica vasculosa lentis TVL and iris hyperaemia were always present at the time of STR-ROP. No eye without TVL received treatment. As good quality anterior segment images were not available for all infants, the overall percentage of eyes with ROP and persistent TVL, and the fraction of those that had STR-ROP could not be evaluated. Examples of persistent TVL are shown in Fig. 3. Imaging of the anterior segment appears important in the assessment of STR-ROP.
Binocular indirect ophthalmoscopy (BIO) versus digital imaging

Although both BIO and WFDI with the RetCam120 were recommended in our study protocol, no systematic documentation of the actual number of dual examinations is available from the NICUs outside Regensburg, nor concerning the order of examinations, i.e. BIO first or digital imaging first. It is noteworthy that none of the peripheral ophthalmologists reported on higher ROP stages with BIO compared to our results from WFDI analysis.

A subset of 41 consecutive infants (63 image sets, 125 eyes) screened in the NICU in Regensburg received dual masked examinations; BIO from an experienced paediatric ophthalmologist (HE), and WFDI from a senior resident. The images were reevaluated in 2008 by the senior ophthalmologist B.L. BW ranged from $560 \mathrm{~g}$ to $1,880 \mathrm{~g}$ (mean $1,357 \mathrm{~g}$, SD $288 \mathrm{~g}$ ), GA from 2433 weeks (mean 30 wks, SD 2 wks). The infants received one to eight examinations (mean 1.6). Agreement was found for 53 examinations, i.e. in $84 \%$. Disagreement was seen in ten examinations (16\%); in five examinations $(8 \%)$, BIO reported a higher stage, and in five examinations (8\%), the RetCam images indicated a higher stage. The highest stage seen in the eyes with disagreement was stage 2 , peripheral zone II, and the largest difference was one stage in zone III. As to the classifications no ROP, CR-ROP and STR-ROP, no change in classification resulted in four of the ten examinations with disagreement $(6.4 \%)$; in three instances, BIO found CR-ROP (stage 1, zone II-III) compared to no ROP with WFDI (4.8\%), and in three instances, WFDI found CR-ROP (stage 1, zone II-III) compared to no ROP with BIO (4.8\%).

\section{Discussion}

Several studies have investigated different issues for the use of the RetCam120 in the management of ROP [32-44, 47, 48]. In an early study by Yen et al., ROP-screening with the RetCam 120 was performed by neonatal nurses in preterm babies at risk [33, 42]. Pictures were taken only at two points of time in the neonatal phase; at a PMA of 3234 weeks, and at 38-40 weeks PMA. WFDI was considered to have an insufficient sensitivity to be recommended as a substitute for BIO in screening for ROP. Contrary to the WFDI, conventional BIO screening was performed according to the actual guidelines, i.e. every 2 weeks. Roth et al. also compared conventional screening with WFDI [34]. The sensitivity to detect stage 1 or 2 ROP in peripheral zone II or III with RetCam120 images was low. However, in those missed cases ROP regressed 
spontaneously. In a study by Schwartz et al. [32], telemedical evaluations and recommendations of WFDI were compared with conventional onsite evaluations and recommendations, to see if the local examiner and the remote examiner would reach the same conclusion for treatment. All but one of the 19 eyes examined had an advanced stage of ROP (at least prethreshold disease). They found agreement with both methods; in one instance, plus disease was correctly identified accurately with WFDI but not with BIO. Following the early rather pessimistic reports by Yen $[33,42]$, the same group has now published a series of papers evaluating in detail sensitivity, specificity, positive and negative predictive value of WFDI and telemedicine for ROP screening [38-40]. In their paper from 2007, they report on sensitivity for type 2 prethreshold or worse ROP at PMA $35-37$ weeks of $85.1 \%$ to $94.3 \%$ for three different, highly experienced graders, and $100 \%$ sensitivity for all three graders for TR-ROP, with a specificity of $80.6 \%$ to $94.1 \%$. In this particular study, only three images were taken as a routine, which is different from the present study. Lately, the Photo-ROP study group has published design issues of their trial, which included establishing the information technology infrastructure for an ROP study based on digital imaging, defining the study endpoints, estimating event rates, defining a standardized imaging protocol, and defining standards for interpretation of image quality and clinical findings [45].

\section{Detection of ROP with WFDI and with BIO}

Our study reflects the typical population of preterm infants that a general ophthalmologist will see in Germany as a consultant in a NICU. Compared to some other studies using conventional screening with BIO, the incidence of CR-ROP $(27.6 \%)$ in our total population of 1,222 premature babies appears comparable to the reported incidence of ROP in similarly immature babies (Table 6). When analyzing only children with a $\mathrm{BW}<1,250 \mathrm{~g}$, the incidence of CR-ROP was
$51.6 \%(253 / 490)$, and went up to $62.2 \%$ in children with a $\mathrm{BW}<1,000 \mathrm{~g}(171 / 275)$. These numbers are comparable to those reported in other series [42-44]. In our work, with a total of 1,222 babies screened between 2001 and 2006, no incidents were noted where TR-ROP was missed with timely RetCam120 examinations. When comparing BIO and RetCam images results in 125 eyes from 63 consecutive examinations screened in the NICU in Regensburg, agreement between the two methods was high, at $84 \%$. Disagreement occurred for both BIO and WFDI reading, reporting a higher stage with the respective method, and thus questioning $\mathrm{BIO}$ as the gold standard. In the cases where BIO reported a higher stage of ROP, the disease was in peripheral zone III. A similar evaluation of the sensitivity was not possible in the NICUs outside Regensburg, due to the study design (which is certainly a limitation). However, it appears highly unlikely that treatable disease was missed, as all infants were followed until retinal vascularisation was complete, i.e. reaching out to mid-peripheral zone III, or any stage of ROP which had resolved spontaneously. Also, none of the local ophthalmologists reported on higher stages of CR-ROP with BIO compared to our image readings, or on undetected ROP at later follow-up. Therefore, we conclude that the sensitivity to detect STR-ROP was $100 \%$ in our study, and the overall positive predictive value to detect TRROP was $88.2 \%$. This is a major achievement compared to $37 \%$ of late referrals (13/35 prematures) we had observed between 1992 and 1997 before starting our telemedical program using WFDI [21].

Recently, Scott et al. [44] reported their results in a larger subset of infants questioning whether BIO should still be considered the gold standard for ROP screening. In fact, in an independent analysis of images from 134 eyes, two physicians had an intraphysician agreement between BIO and WFDI reading of $86.7 \%$ and $85.4 \%$ respectively. The $13.6 \%$ of misinterpretations were false negatives from BIO misjudging zone I, plus disease and mild ROP, and corroborates with our own findings. On the other hand,

Table 6 Comparative data of incidence of ROP in infants at risk detected by screening with BIO and RetCam120 imaging

\begin{tabular}{|c|c|c|c|c|c|}
\hline Study & Infants \# & Mean GA (wks) & Mean BW (g) & ROP (all stages) & Method \\
\hline Palmer et al. 1991 [49] & 4,099 & & All $<1,250$ g ! & $65.8 \%$ & $\mathrm{BIO}$ \\
\hline Larsson et al. 2002* [50] & 253 & 28.5 & 1,118 & $36.4 \%$ & $\mathrm{BIO}$ \\
\hline Larsson et al. $2002 * *$ [51] & 392 & 29.4 & 1,381 & $25.5 \%$ & $\mathrm{BIO}$ \\
\hline Mathew et al. 2002 [52] & 205 & 28 & 1,205 & $31.2 \%$ & $\mathrm{BIO}$ \\
\hline Chiang et al. 2004 ${ }^{\#}$ [48] & 10,596 & Not indicated & 1,420 & $24 \%$ & $\mathrm{BIO}$ \\
\hline This study+ & 1,222 & 30 & 1,395 & $27.6 \%$ & RetCam 120 \\
\hline
\end{tabular}

*Born between 1998-2000, BW < 1500g, **born between 1998-2000, GA < 32 wks, \# born between 1996-2000 with length of stay > 28 days; ROP incidence in dependence on mean BW given for infants born in 2000.. + Clinical relevant ROP CR-ROP. CR-ROP includes all stages in zone I and II as well as in central zone III. Peripheral zone III and ora serrata usually cannot be imaged with the RetCam120. The much higher incidence of acute ROP in the Cryo-ROP study is explained by the lower gestational age. The incidence in the present study may be due to significant improvements in neonatal care since 1991 and to the fact that in our study only CSROP was included. 
caution is still recommended, in particular in aggressive posterior ROP, as there is at least one observation that WFDI readings only 10 minutes apart showed very different degrees of severity [47].

\section{STR-ROP versus RW-ROP}

Ells and coworkers analysed the safety of telemedical classification of referral-warranted ROP RW-ROP [36]. They included all zone I disease, plus and stage 3 disease. In contrast, we defined STR-ROP as the stage we wanted to see referred for a BIO to take the final treatment decision. We chose this narrower definition to avoid unnecessary referrals as much as possible, but without increasing the risk for late referrals. Comparing the number of eyes with RW-ROP and with STR-ROP to eyes that finally received laser therapy in the two series, the different referral pattern becomes clear. Only $43.5 \%$ of eyes with RW-ROP (10/23 eyes) received laser therapy in the study by Ells, compared to $88.2 \%$ of the eyes with STR-ROP in the current study (30/34). The wider definition of RW-ROP by Ells may contain a higher level of safety. At the same time, this definition considerably lowers the positive predictive value as to TR-ROP. A high positive predictive value is, however, highly desirable to minimize the number of unnecessary transferrals of prematures that are stressful, hence potentially harmful, and also costly.

\section{Limitations of WFDI}

There is no doubt that DMFI faces limitations, just as examination with BIO does. In agreement with other authors $[34,35,37]$, we consider image quality with the RetCam 120 to be suboptimal, in particular, for peripheral nasal zone II and peripheral zone III. Technical improvements to enhance image quality of peripheral retinal parts would be highly desirable. When using additional criteria such as degree of peripheral vessel arborisation and vessel diameter for image assessment, we were able to limit the number of infants whose retina could not be evaluated. We report that only $2.4 \%$ of the imaging sessions did not have sufficient image quality to assess the actual risk. This was not a major problem, as repeat examination yielded images with sufficient quality. In our experience, all users learned how to image after being instructed on only a few babies. Obtaining adequate pupillary dilation was crucial. In the case of insufficient image quality, we ordered the exam to be repeated within a week or less according to disease severity and degree of maturity of the infant. At follow-up, none of the infants with non-readable images at the initial exam developed any relevant ROP. Very small infants can be difficult to image peripherally; however, in most of these tiny babies at least the posterior pole can be imaged, and this is generally sufficient for risk assessment, if follow-up examinations are advised until vascularisation is complete in mid-peripheral zone III. There is also skill involved in reading the images. The stereo view of the BIO is missing, but is being replaced by learning to read subtle signs at the border of vascularisation. It is essential that experts who are familiar with both the funduscopic aspect with BIO and the appearance with the RetCam120 read the images, in order not to confuse stages 2 and 3 and peripheral stage 4 . In this respect we would like to emphasize the importance of continuing to train young ophthalmologists with BIO to diagnose acute ROP. With these precautions, WFDI is a valuable screening tool.

Acceptance of our study by neonatologists and parents

Although no systematic survey of the acceptance has been conducted in this study, all 15 neonatologists directly involved in the study emphasized the advantage of having images of the retinal changes. They also felt that WFDI and image evaluation at the Reading Centre minimized the risk of blindness in the infants. All neonatologists stated that this outweighed the disadvantage of the longer time required for the exam of the infants. Examination time was highly examiner-dependent, and was only about 5 minutes per infant in the NICU in Regensburg, where imaging was performed by trained paediatric ophthalmologists of the Reading Centre. In the peripheral NICUs, paediatricians usually helped the ophthalmologists to code and collect the images, and to transmit the coded images via ISDN (phone line) and the coded paediatric data by fax or email. The parents' compliance was evaluated from their consent to participate in the telemedicine program. Once detailed information had been provided by the neonatologist, virtually all parents consented to the telemedicine program. Only parents who had consented were included in the study.

Limitations of our study

The major limitation of our study is that comparison of WFDI and BIO was performed in only a fraction of infants. A comparison in all infants did not appear feasible, as in the peripheral NICUs screening was always performed by single ophthalmologists. So it cannot be excluded that WFDI was done after BIO, and that therefore the examiner was biased as to the result. A significant influence appears, however, unlikely given the limited quality of many image sets and the fact that BIO was - contrary to the protocol-not always added; this was reported by the paediatricians who helped with the examination. Bias was also minimized as the results of the local ophthalmologist's examination were unknown to the readers at the Reading Centre, who based their diagnosis exclusively on reading of the images. 
Cost-effectiveness of WFDI-based telemedicine

A recent study has looked into the cost-utility relationship of telemedicine with digital imaging compared to BIO [53]. Although such an evaluation is difficult due to the enormous complexity of factors and aspects involved, it would appear that telemedicine can be less expensive than BIO based on US rates of reimbursement. No formal analysis on the cost-effectiveness of our program has been done so far. However, given 100\% sensitivity to detect STR-ROP, and comparing this to $37 \%$ late referrals to the Department of Ophthalmology at the University of Regensburg between 1992 and 1997, with consecutive blindness in $62 \%$ of the infants (8/13) and unilateral blindness in $38 \%(5 / 13)$, the benefit of our program, both from an economic and a medical standpoint would appear evident. However, caution is necessary when comparing incidence of very advanced ROP and treatment outcome in infants from two completely different time periods.

\section{Conclusion}

We demonstrate the use of WFDI to screen for CR-ROP in a routine clinical telemedical setting with ophthalmologists taking the images. At present, nurses or professional photographers are not using WFDI in Germany. With a documented sensitivity of $100 \%$ to detect STR-ROP and a positive predictive value of $88.2 \%$ for TR-ROP in a large cohort of 913 babies in four different peripheral NICUs, this approach is shown to be a safe and effective method to timely detect potentially blinding ROP, and compares favourably to the high percentage of late referrals we had seen before starting our telemedical program. We get support from a number of recent studies that have compared BIO with WFDI in several studies, yet with much smaller sample sizes. The weakness of our study is that no formal comparison of BIO and WFDI is available for the majority of the exams. Key to success are highly qualified image readers that have to be experienced with $\mathrm{BIO}$ as well. Beyond this, our database combining all relevant paediatric data with serial imaging of all prematures at risk for ROP also allows re-evaluating classification of TR-ROP according to evolving treatment indications, and provides an extraordinary resource for further investigations and research.

\footnotetext{
Acknowledgments We thank all families who participated in the screening program.

Participants of the Cooperative Study Group

Neonatal Intensive Care Units:

Kinderklinik Bayreuth, D-95445 Bayreuth (Head: Prof. Dr. G.F.
}

Wündisch, Prof. Dr. T. Rupprecht): Dr. T. Fischer, Dr. B. BrummerGugel, Dr. T. Rauch, Dr. M. Muzzolini, Dr. A. Birkenmeier, Dr. W. Pohl; Kinderklinik Deggendorf, D-94469 Deggendorf (Head: Dr. H. Arends, Dr. M. Mandl): Dr. M. Welsch;

Kinderklinik Dritter Orden, D-94037 Passau (Head: Prof. Dr. F. Staudt): Dr. C. Schmidtlein;

Klinik für Kinder- und Jugendmedizin, St. Hedwig, D-93049 Regensburg, (Head: Prof. Dr. H. Segerer);

Kinderklinik am Klinikum Weiden, D- 92637 Weiden (Head: Dr. E. Lachmann): Dr. K. Adam, Dr. P. Gutdeutsch, Dr. H. Madlon. General ophthalmologists:

D-95444 Bayreuth: PD Dr. W. Schrems, Dr. E. Glaab-Schrems D-94469 Deggendorf: Dr. M. Kranewitter

D-94032 Passau: Dr. N. Demmler, Dr. M. Demmler-Hackenberg, Dr. C. von Düring, Dr. D. Liebsch, Dr. C. Tacke, PD. Dr. T.-M. Wohlrab D-92637 Weiden: Dr. D. Lux/Dr. A. Rupprecht, Dr. H. Hößler

\section{Reading and Study Centre:}

Dept. of Paediatric Ophthalmology, Strabismology and Ophthalmogenetics, Klinikum, University of Regensburg: Prof. Dr. B. Lorenz (Head), Dr. C. Brückner, Dr. H. Elflein, Dr. F. Gora, Dr. I. OberacherVelten, Dr. M. Preising, Dr. P. Roth, Dipl-Biol. N. Schneider, Dr. K. Spasovska, Dr. B. Wabbels, Dr. E. Wegscheider

Bayerisches Forschungszentrum für Wissensbasierte Systeme (ForWiss):

Universität Passau: Prof. Dr. E. Fuchs, M. Donaubauer, PD Dr. G. Pisinger

Open Access This article is distributed under the terms of the Creative Commons Attribution Noncommercial License which permits any noncommercial use, distribution, and reproduction in any medium, provided the original author(s) and source are credited.

\section{References}

1. Cooke RW, Clark D, Hickey-Dwyer M, Weindling AM (1993) The apparent role of blood transfusions in the development of retinopathy of prematurity. Eur J Pediatr 152:833-836, doi:10.1007/BF02073381

2. Bossi E, Koerner F, Flury B, Zulauf M (1984) Retinopathy of prematurity: a risk factor analysis with univariate and multivariate statistics. Helv Paediatr Acta 39:307-317

3. Brown DR, Biglan AW, Stretavsky MM (1990) Retinopathy of prematurity: the relationship with intraventricular hemorrhage and bronchopulmonary dysplasia. J Pediatr Ophthalmol Strabismus 27:268-271

4. Flynn JT (1983) Acute proliferative retrolental fibroplasia: multivariate risk analysis. Trans Am Ophthalmol Soc 81:549-591

5. Flynn JT, Bancalari E, Snyder ES, Goldberg RN, Feuer W, Cassady J, Schiffman J, Feldman HI, Bachynski B, Buckley E (1992) A cohort study of transcutaneous oxygen tension and the incidence and severity of retinopathy of prematurity. N Engl J Med 326:1050-1054

6. Gallo JE, Jacobson L, Broberger U (1993) Perinatal factors associated with retinopathy of prematurity. Acta Paediatr 82:829-834

7. Hesse L, Eberl W, Schlaud M, Poets CF (1997) Blood transfusion. Iron load and retinopathy of prematurity. Eur J Pediatr 156:465470, doi:10.1007/s004310050641

8. Holmström G, Broberger U, Thomassen P (1998) Neonatal risk factors for retinopathy of prematurity - a population-based study. Acta Ophthalmol Scand 76:204-207, doi:10.1034/j.16000420.1998.760216.x

9. Hellström A, Perruzzi C, Ju M, Engström E, Hard AL, Liu JL, Albertsson-Wikland K, Carlsson B, Niklasson A, Sjodell L, LeRoith 
D, Senger DR, Smith LE (2001) Low IGF-I suppresses VEGFsurvival signaling in retinal endothelial cells: direct correlation with clinical retinopathy of prematurity. Proc Natl Acad Sci USA 98:5804-5808, doi:10.1073/pnas.101113998

10. Hellström A, Engström E, Hard AL, Albertsson-Wikland K, Carlsson B, Niklasson A, Lofqvist C, Svensson E, Holm S, Ewald U, Holmström G, Smith LE (2003) Postnatal serum insulin-like growth factor I deficiency is associated with retinopathy of prematurity and other complications of premature birth. Pediatrics 112:1016-1020, doi:10.1542/peds.112.5.1016

11. Inder TE, Clemett RS, Austin NC, Graham P, Darlow BA (1997) High iron status in very low birth weight infants is associated with an increased risk of retinopathy of prematurity. J Pediatr 131:541544, doi:10.1016/S0022-3476(97)70058-1

12. Prendiville A, Schulenburg WE (1988) Clinical factors associated with retinopathy of prematurity. Arch Dis Child 63:522-527, doi:10.1136/adc.63.5.522

13. Sacks LM, Schaffer DB, Anday EK, Peckham GJ, ivoriaPapadopoulos M (1981) Retrolental fibroplasia and blood transfusion in very low-birth-weight infants. Pediatrics 68:770-774

14. Gilbert C, Rahi J, Eckstein M, O'Sullivan J, Foster A (1997) Retinopathy of prematurity in middle-income countries. Lancet 350:12-14, doi:10.1016/S0140-6736(97)01107-0

15. Gilbert C, Fielder A, Gordillo L, Quinn G, Semiglia R, Visintin P, Zin A (2005) Characteristics of infants with severe retinopathy of prematurity in countries with low, moderate, and high levels of development: implications for screening programs. Pediatrics 115: e518-e525, doi:10.1542/peds.2004-1180

16. The Committee for the Classification of Retinopathy of Prematurity (1984) An international classification of retinopathy of prematurity. Arch Ophthalmol 102:1130-1134

17. Cryotherapy for Retinopathy of Prematurity Cooperative Group (1990) Multicenter trial of cryotherapy for retinopathy of prematurity. Three-month outcome. Cryotherapy for Retinopathy of Prematurity Cooperative Group. Arch Ophthalmol 108: 195-204

18. Banach MJ, Berinstein DM (2001) Laser therapy for retinopathy of prematurity. Curr Opin Ophthalmol 12:164-170, doi:10.1097/ 00055735-200106000-00003

19. Seiberth V, Linderkamp O, Vardarli I, Knorz MC, Liesenhoff $\mathrm{H}$ (1996) Diodenlaserkoagulation der Retinopathia praematurorum Stadium 3+. Ophthalmologe 93:182-189

20. Palmer EA (1990) Results of U.S. randomized clinical trial of cryotherapy for ROP (CRYO-ROP). Doc Ophthalmol 74:245251, doi:10.1007/BF02482615

21. Lorenz B, Bock M, Müller HM, Massie NA (1999) Telemedicine based screening of infants at risk for retinopathy of prematurity. Stud Health Technol Inform 64:155-163

22. Early Treatment For Retinopathy Of Prematurity Cooperative Group (2003) Revised indications for the treatment of retinopathy of prematurity: results of the early treatment for retinopathy of prematurity randomized trial. Arch Ophthalmol 121:1684-1694, doi:10.1001/archopht.121.12.1684

23. Good WV, Hardy RJ (2001) The multicenter study of Early Treatment for Retinopathy of Prematurity (ETROP). Ophthalmology 108:1013-1014, doi:10.1016/S0161-6420(01)00540-1

24. Joint statement of the American Academy of Pediatrics tAAfPOaSatAAoO (1997) Screening examination of premature infants for retinopathy of prematurity. Ophthalmology 104:888-889

25. American Academy of Pediatrics Retinopathy of Prematurity Subcommittee (2001) Screening examination of premature infants for retinopathy of prematurity. Pediatrics 108:809-811, doi:10.1542/peds.108.3.809

26. Policy Statement of the Section on Ophthalmology AAoPAAoOAafpOaS (2006) Screening examination of premature infants for retinopathy of prematurity. Pediatrics 117:572-576, doi:10.1542/peds.2005-2749
27. Joint Working Party of The Royal College of Ophthalmologists and the British Association of Perinatal Medicine (1996) Retinopathy of prematurity: guidelines for screening and treatment. The report of a Joint Working Party of The Royal College of Ophthalmologists and the British Association of Perinatal Medicine. Early Hum Dev 46:239-258, doi:10.1016/S0378-3782(96)01747-1

28. Wilkinson AR, Haines L, Head K, Fielder AR (2008) UK retinopathy of prematurity guideline. Early Hum Dev 84:71-74, doi:10.1016/j.earlhumdev.2007.12.004

29. Clemens S, Eckardt C, Gerding H, Grote A, Jandeck C, Kellner U, Lorenz B, Petersen J, Seiberth V, Stark N, Ulbig MW, Zubcov A, Jorch G, Pohlandt F (1999) Augenärztliche ScreeningUntersuchung von Frühgeborenen. Ophthalmologe 96:257-263, doi: $10.1007 / \mathrm{s} 003470050396$

30. Jandeck C, Kellner U, Lorenz B, Seiberth V (2008) Leitlinie zur augenarztlichen Screening-Untersuchung von FruhgeborenenArbeitsgruppe der Retinologischen Gesellschaft zur Erstellung der Leitlinie zur augenarztlichen Screening-Untersuchung von Fruhgeborenen. Klin Monatsbl Augenheilkd 225:123-130, doi:10.1055/s-2008-1027168

31. Reynolds JD (2001) The management of retinopathy of prematurity. Paediatr Drugs 3:263-272, doi:10.2165/00128072-20010304000003

32. Schwartz SD, Harrison SA, Ferrone PJ, Trese MT (2000) Telemedical evaluation and management of retinopathy of prematurity using a fiberoptic digital fundus camera. Ophthalmology 107:25-28, doi:10.1016/S0161-6420(99)00003-2

33. Yen KG, Hess D, Burke B, Johnson RA, Feuer WJ, Flynn JT (2000) The optimum time to employ telephotoscreening to detect retinopathy of prematurity. Trans Am Ophthalmol Soc 98:145-151

34. Roth DB, Morales D, Feuer WJ, Hess D, Johnson RA, Flynn JT (2001) Screening for retinopathy of prematurity employing the RetCam 120: sensitivity and specificity. Arch Ophthalmol 119:268-272

35. Seiberth V, Woldt C (2001) Weitwinkelfundusdokumentation bei Retinopathia praematurorum. Ophthalmologe 98:960-963, doi:10.1007/s003470170044

36. Ells AL, Holmes JM, Astle WF, Williams G, Leske DA, Fielden M, Uphill B, Jennett P, Hebert M (2003) Telemedicine approach to screening for severe retinopathy of prematurity: a pilot study. Ophthalmology 110:2113-2117, doi:10.1016/S0161-6420(03) 00831-5

37. Wu C, Petersen RA, VanderVeen DK (2006) RetCam imaging for retinopathy of prematurity screening. J AAPOS 10:107-111, doi:10.1016/j.jaapos.2005.11.019

38. Chiang MF, Starren J, Du YE, Keenan JD, Schiff WM, Barile GR, Li J, Johnson RA, Hess DJ, Flynn JT (2006) Remote image based retinopathy of prematurity diagnosis: a receiver operating characteristic analysis of accuracy. Br J Ophthalmol 90:1292-1296, doi:10.1136/bjo.2006.091900

39. Chiang MF, Keenan JD, Starren J, Du YE, Schiff WM, Barile GR, Li J, Johnson RA, Hess DJ, Flynn JT (2006) Accuracy and reliability of remote retinopathy of prematurity diagnosis. Arch Ophthalmol 124:322-327, doi:10.1001/archopht.124.3.322

40. Chiang MF, Wang L, Busuioc M, Du YE, Chan P, Kane SA, Lee TC, Weissgold DJ, Berrocal AM, Coki O, Flynn JT, Starren J (2007) Telemedical retinopathy of prematurity diagnosis: accuracy, reliability, and image quality. Arch Ophthalmol 125:1531-1538, doi:10.1001/archopht.125.11.1531

41. Fielder AR, Cocker KD, Capone A Jr, Trese MT (2002) Screening for retinopathy of prematurity using wide-field digital retinal imaging: sensitivity and specificity. Arch Ophthalmol 120:1234

42. Yen KG, Hess D, Burke B, Johnson RA, Feuer WJ, Flynn JT (2002) Telephotoscreening to detect retinopathy of prematurity: preliminary study of the optimum time to employ digital fundus 
camera imaging to detect ROP. J AAPOS 6:64-70, doi:10.1067/ mpa.2002.121616

43. Sommer C, Gouillard C, Brugniart C, Talmud M, Bednarek N, Morville P (2003) Dépistage et suivi de la rétinopathie du prematuré par caméra de rétine (Retcam 120) : expérience d'une équipe de néonatalogistes à propos de 145 cas. Arch Pediatr 10:694-699, doi:10.1016/S0929-693X(03)00295-1

44. Scott KE, Kim DY, Wang L, Kane SA, Coki O, Starren J, Flynn JT, Chiang MF (2008) Telemedical diagnosis of retinopathy of prematurity intraphysician agreement between ophthalmoscopic examination and image-based interpretation. Ophthalmology 115:1222-1228, doi:10.1016/j.ophtha.2007.09.006

45. Balasubramanian M, Capone A Jr, Hartnett ME, Pignatto S, Trese MT (2006) The Photographic Screening for Retinopathy of Prematurity Study (Photo-ROP): study design and baseline characteristics of enrolled patients. Retina 26:S4-S10, doi:10.1097/01.iae.0000244291.09499.88

46. Kemper AR, Wallace DK, Quinn GE (2008) Systematic review of digital imaging screening strategies for retinopathy of prematurity. Pediatrics 122:825-830, doi:10.1542/peds.2007-3667

47. Koreen S, Lopez R, Jokl DH, Flynn JT, Chiang MF (2008) Variation in appearance of severe zone 1 retinopathy of prematurity during wide-angle contact photography. Arch Ophthalmol 126:736-737, doi:10.1001/archopht.126.5.736

48. Chiang MF, Arons RR, Flynn JT, Starren JB (2004) Incidence of retinopathy of prematurity from 1996 to 2000: analysis of a comprehensive New York state patient database. Ophthalmology 111:1317-1325, doi:10.1016/j.ophtha.2003.10.030

49. Palmer EA, Flynn JT, Hardy RJ, Phelps DL, Phillips CL, Schaffer DB, Tung B (1991) Incidence and early course of retinopathy of prematurity. The Cryotherapy for Retinopathy of Prematurity Cooperative Group. Ophthalmology 98:1628-1640

50. Larsson E, Carle-Petrelius B, Cernerud G, Ots L, Wallin A, Holmström G (2002) Incidence of ROP in two consecutive Swedish population based studies. Br J Ophthalmol 86:11221126, doi:10.1136/bjo.86.10.1122

51. Larsson E, Holmström G (2002) Screening for retinopathy of prematurity: evaluation and modification of guidelines. $\mathrm{Br} \mathrm{J}$ Ophthalmol 86:1399-1402, doi:10.1136/bjo.86.12.1399

52. Mathew MR, Fern AI, Hill R (2002) Retinopathy of prematurity: are we screening too many babies? Eye 16:538-542, doi:10.1038/ sj.eye. 6700031

53. Jackson KM, Scott KE, Graff ZJ, Bateman DA, Flynn JT, Keenan JD, Chiang MF (2008) Cost-utility analysis of telemedicine and ophthalmoscopy for retinopathy of prematurity management. Arch Ophthalmol 126:493-499, doi:10.1001/ archopht.126.4.493

54. Lorenz B (2006) Screening for Retinopathy of Prematurity. In: Lorenz B, Moore AT (eds) Pediatric Ophthalmology, NeuroOphthalmology, Genetics. (5) in Essentials in Ophthalmology ed. Krieglstein GK and Weinreb RN. Springer, Berlin, pp 63-110. ISBN: 3-540-22594-3 